\title{
Protective Effect of Phillyrin on Lethal LPS-Induced Neutrophil Inflammation in Zebrafish
}

\author{
Liling Yanga,b Xiangjun Zhou ${ }^{\mathrm{a}}$ Weijuan Huang ${ }^{\mathrm{a}}$ Qin Fang ${ }^{\mathrm{b}}$ Jianlan $\mathrm{Hu}^{\mathrm{b}}$ \\ Linzhong Yu ${ }^{b}$ Ning Mad Wenqing Zhang ${ }^{d}$
}

a'Department of Pharmacy, The Dongguan Affiliated Hospital of Medical college of Jinan University, The Fifth People's Hospital of Dongguan, Dongguan, 'bSchool of Traditional Chinese Medicine, Southern Medical University, Guangzhou, 'School of Pharmacy, Guangdong Medical University, Dongguan, 'Key Laboratory of Zebrafish Modeling and Drug Screening for Human Diseases of Guangdong Higher Education Institutes, Department of Cell Biology, Southern Medical University, Guangzhou, China

\section{Key Words}

Lps • Phillyrin • Neutrophils • Inflammation • Zebrafish • MyD88 • NF-kB

\begin{abstract}
Background/Aims: Forsythia suspensa Vahl. (Oleaceae) fruits are widely used in traditional Chinese medicine to treat pneumonia, typhoid, dysentery, ulcers and oedema. Antibacterial and anti-inflammatory activities have been reported for phillyrin (PHN), the main ingredient in Forsythia suspensa Vahl fruits, in vitro. However, the underlying mechanisms in vivo remain poorly defined. In this study, we discovered that PHN exerted potent anti-inflammatory effects in lethal LPS-induced neutrophil inflammation by suppressing the MyD88-dependent signalling pathway in zebrafish. Methods: LPS-yolk microinjection was used to induce a lethal LPS-infected zebrafish model. The effect of PHN on the survival of zebrafish challenged with lethal LPS was evaluated using survival analysis. The effect of PHN on neutrophil inflammation grading in vivo was assessed by tracking neutrophils with a transgenic line. The effects of PHN on neutrophil production and migration were analysed by $\mathrm{SB}^{+}$cell counts during consecutive hours after modelling. Additionally, key cytokines and members of the MyD88 signalling pathway that are involved in inflammatory response were detected using quantitative RT-PCR. To assess gene expression changes during consecutive hours after modelling, the IL-1 $\beta$, IL-6, TNF- $\alpha$, MyD88, TRIF, ERK1/2, JNK, IKBa and NF-KB expression levels were measured. Results: PHN could protect zebrafish against a lethal LPS challenge in a dose-dependent manner, as indicated by decreased neutrophil infiltration, reduced tissue necrosis and increased survival rates. Up-regulated IL-1 $\beta$, IL- 6 and TNF- $\alpha$ expression also showed the same tendencies of depression by PHN. Critically, PHN significantly inhibited the LPS-induced activation of MyD88, IKBa, and NF-KB but did not affect the expression of ERK1/2 MAPKs or JNK MAPKs in LPSstimulated zebrafish. Additionally, PHN regulated the MyD88/IKB $\alpha / N F-\kappa B$ signalling pathway

L. Yang, X. Zhou and W. Huang contributed equally to this work.




\section{Cellular Physiology Cell Physiol Biochem 2017;43:2074-2087

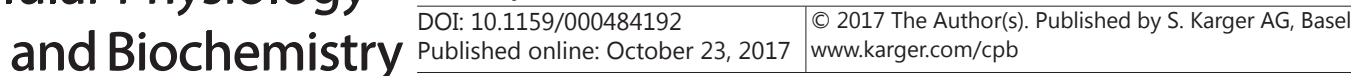 \\ Yang et al.: Protective Effect of PHN in Zebrafish}

by controlling IKB $\alpha$, IL-1 $\beta$, IL-6, and TNF- $\alpha$ expression. Conclusion: This study provides a rationale for the clinical application of PHN as an anti-inflammatory agent.

(C) 2017 The Author(s)

Published by S. Karger AG, Basel

\section{Introduction}

LPS, the major outer membrane constituent of all gram-negative bacteria and their main causative agent, activates a variety of immunocytes, including neutrophils, macrophages and endothelial cells [1], and contributes to the systemic change known as disseminated intravascular coagulation (DIC), systemic inflammatory response syndrome (SIRS) and endotoxic shock $[2,3]$. Neutrophils are key effectors of innate immune response, and LPS has multiple effects on neutrophils, such as inhibiting their apoptosis and promoting their necrosis [4-6]. Studies have demonstrated that LPS transmembrane signalling is initiated by Toll-like receptors (TLRs), which are present in the plasma membranes and endosomes of leukocytes [7]. TLRs recognize LPS and transmit extracellular activation signals inside the cell via the myeloid differentiation protein 88 (MyD88)-dependent pathway or the TIR domaincontaining adaptor inducing IFN- $\beta$ (TRIF)-dependent pathway. The MyD88-dependent response occurs upon TLR dimerization, and its primary effect is activating the nuclear factor $\kappa B(N F-\kappa B)$ and mitogen-activated protein kinase (MAPK) pathways, which include extracellular signal-regulated kinases1/2 (ERK1/2) and c-Jun NH2-terminal kinase (JNK). The MyD88 response produces numerous inflammatory cytokines, including interleukin- $1 \beta$ (IL-1 $\beta$ ), interleukin 6 (IL-6), and tumour necrosis factor-alpha (TNF- $\alpha$ ). The TRIF-dependent pathway leads to the IFN regulatory factor 3/7 (IRF3/7)-mediated expression of type I IFNs (IFN-a and IFN-b) to produce antiviral responses and IFN-inducible chemokines [8-11].

Zebrafish (Danio rerio) hold considerable promise as a drug screening model. Their short generation time, low cost, high clutch-size at the embryonic and larval stages, and optical transparency permit the visualization of pathogens and lesions in real time [12]. Additionally, the zebrafish immune system is remarkably similar to that of mammals [1315]. The main reason for using zebrafish as a vertebrate model for studying and screening antiendotoxic drugs is that they serve as an advanced tool for genomic and large-scale transgenic line analysis [16]. For example, the biological behaviour of larvae neutrophils can be detected in real time using the MPO:GFP transgenic line [17], whereas such detection is unachievable with vertebrate species.

Forsythia suspensa Vahl (Oleaceae), a climbing plant, is widely distributed throughout China, Korea and Japan. The fruit of this plant is a well-known traditional Chinese medicine (TCM) named 'Lianqiao' in Chinese. More than 40 Chinese medicinal preparations containing F. suspansa are listed in the Chinese Pharmacopoeia [18]. The crude drug has been widely used as antipyretic, antidotal and anti-inflammatory agents for the treatment of infections, including pneumonia, typhoid, dysentery, ulcers and oedema [19, 20]. Moreover, crude extracts have been shown to suppress vomiting; resist hepatic injury; inhibit elastase activity; alleviate hypersensitivity; and exhibit diuretic, antibacterial, antiviral, anti-inflammatory, antioxidant and analgesic effects [21-24]. Phenylethanol glycosides, lignans and flavonoids were proven to be responsible for the various biological activities of the herb, and they were all isolated from the title plant [25]. Phillyrin (PHN) (Fig. 1), a lignin, plays important roles in the antioxidant, antibacterial, anti-obesity and anti-inflammatory effects of Lianqiao [26-28]. Recently, an in vitro study reported that lignans from the flowers of $O$. fragrans

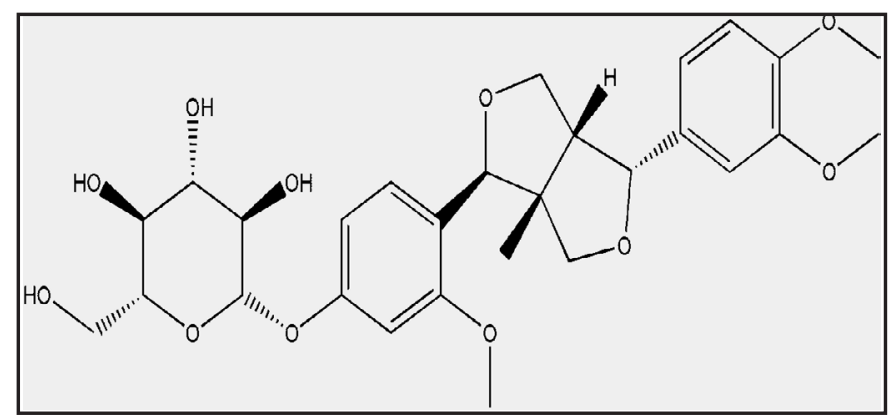

Fig. 1. Chemical structure of phillyrin (PHN). 
var. aurantiacus could inhibit the release of nitric oxide in RAW 264.7 macrophages [29]. However, the underlying in vivo mechanisms remain poorly defined.

In this study, we first found that PHN exerted antiendotoxin inflammatory effects via diminishing the expression of Myd88, which led to inhibition of the I $\mathrm{Ba} / \mathrm{NF}-\kappa \mathrm{B}$ pathway but not of the ERK1/2 MAPKs or JNK MAPKs pathways, in zebrafish. Our research provides scientific evidence for the therapeutic application of PHN.

\section{Materials and Methods}

\section{Materials}

1-phenyl-2-thiourea (PTU), LPS (E. coli, 055: B5, L2880, total impurities <3\% protein), and Sudan Black B (SB) were purchased from Sigma-Aldrich Co. (St Louis, MO, USA); Bortezomiob (PS341) was purchased from Ben Venue Laboratories Inc. (USA); RNAlater ${ }^{\circledR}$ solution was obtained from Ambion Inc. (Austin, TX, USA). RNAiso Plus, the PrimeScript ${ }^{\circledR}$ RT Reagent kit and the SYBR Green PCR Core Reagent kit were purchased from TaKaRa Biotechnology Co. (Japan). The PCR primers were purchased from Invitrogen (USA). PHN was purchased from the Guangdong Institute for Drug Control (HPLC $>98 \%$, China). The other chemicals and reagents used were obtained in the highest grade commercially available.

\section{Zebrafish embryo and larvae maintenance}

Zebrafish embryos were maintained and raised according to the protocol described by Westerfield [30]. The transgenic line Tg (MPO:GFP) [17] was kindly provided by the Key Laboratory of Zebrafish Modeling and Drug Screening for Human Diseases Institute at Southern Medical University (Guangzhou, China). All the experimental protocols and animals used in this research were approved by the Ethical Committee of Southern Medical University.

\section{Acute inflammatory model and drug administration}

Microinjection (Narshige PLI-100) was performed in a 2-nl volume per larva (except for the normal control). Three days post-fertilization (dpf), zebrafish yolks were injected with $0.5 \mathrm{mg} / \mathrm{ml} \mathrm{LPS} \mathrm{to} \mathrm{induce} \mathrm{the}$ lethal endotoxin-infected model [31]. Phosphate-buffered saline (PBS) was used as the negative control. Before administration to determine PHN effects, PHN (1.0, 3.3, $10.0 \mu \mathrm{g} / \mathrm{ml}$ ), dexamethasone (DEX, 5.0 $\mu \mathrm{g} / \mathrm{ml}$ ) (positive control) and PS341 (a IкB $\alpha$ degradation inhibitor, $10.0 \mu \mathrm{g} / \mathrm{ml}$ ) were dissolved in $0.1 \%$ dimethyl sulfoxide (DMSO) in egg water at the required concentrations. After recovering from anaesthesia $(0.02 \%$ tricaine), larvae from each group were moved to a 6 -well plate. The treatment groups were exposed to PHN, DEX or PS341. All the groups were maintained in a final volume of $5 \mathrm{ml}$ at $28.5^{\circ} \mathrm{C}$. The fish were evaluated for mortality and disease symptoms for $96 \mathrm{~h}$ post-injection (hpi).

\section{Inflammation assessment criteria}

The transgenic Tg (MPO:GFP) line was used so that neutrophils could be detected by green fluorescence in real time. Neutrophil recruitment was evaluated by Tg (MPO:GFP) with fluorescently marked cell populations in vivo after injecting the yolks with LPS. The neutrophil recruitment grade was assessed at 12 and $24 \mathrm{hpi}$, and the assessment criteria were as follows: Grade $1=$ normal (zero or several neutrophils, no necrosis); Grade 2 = neutrophils scattered throughout the yolk, no necrosis; Grade 3 = neutrophil recruitment to the LPS site, no necrosis; Grade $4=$ bulk infiltration of neutrophils and neutrophil adhesion, no necrosis; Grade 5 = yolk deformation and necrosis.

\section{SB Staining}

Zebrafish larva yolks injected with $0.5 \mathrm{mg} / \mathrm{ml}$ LPS at $3 \mathrm{dpf}$ were collected at 1, 3, 6, 12, and 24 hpi for SB staining. Negative control, positive control and PHN treatment larvae were sampled at identical time points. Samples included 10 larvae per time point per treatment. SB staining was performed according to the protocol described by Guyader et al. (2008)[32]. Under a stereomicroscope, $\mathrm{SB}^{+}$cells in yolks and abdominal aortas (AOs) were counted after staining.

Real-time quantitative PCR

Zebrafish larva yolks injected with $0.5 \mathrm{mg} / \mathrm{ml}$ LPS at $3 \mathrm{dpf}$ were collected at 6, 12, 18, and 24 hpi for the extraction of total RNA. Larvae from the other groups were sampled at identical time points. Samples 
included 30 larvae per time point per treatment. Larvae were stored in RNA stabilization solution at $4{ }^{\circ} \mathrm{C}$ until samples from all the time points were collected. Total RNA was extracted using RNAiso Plus according to the manufacturer's instructions. The RNAs were then quantified by UV spectrophotometry (Thermo, NanoDrop 2000). Transcribed cDNAs were then amplified using the Prime Script ${ }^{\circledR}$ RT reagent kit according to the manufacturer's instructions. Real-time quantitative PCR was performed according to the manufacturer's instructions (SYBR Green PCR Core Reagent kit). The primer sequences used were as follows:

$\beta$-actin 130 bps $\{$ F $\} 5^{\prime}$-ATGGATGAGGAAATCGCTG-3',

\{R\} 5'-ATGCCAACCATCACTCCCTG-3';

TNF- $\alpha 139$ bps $\{$ F $\} 5^{\prime}$-GCTGGATCTTCAAAGTCGGGTGTA-3',

$\{\mathrm{R}\} 5^{\prime}$-TGTGAGTCTCAGCACACTTCCATC-3';

IL-1 $\beta 124$ bps $\{$ F $\} 5^{\prime}$-TGGACTTCGCAGCACAAAATG-3',

$\{$ R $\} 5^{\prime}$-GTTCACTTCACGCTCTTGGATG-3';

IL-6 136 bps $\{$ F $\} 5^{\prime}$-AGACCGCTGCCTGTCTAAAA-3',

\{R\} 5'-TTTGATGTCGTTCACCAGGA-3';

MyD88 116 bps \{F\} 5'-GAGGATGGTGGTGGTCATCT-3'

$\{\mathrm{R}\} 5^{\prime}$-CGACAGGGATTAGCCGTTTA-3';

TRIF 141 bps $\{$ F $\} 5^{\prime}$ - TGGGTCAGTTTCCAAGTTCC - $3^{\prime}$

$\{\mathrm{R}\} 5^{\prime}$ - CCACCTTCTGCCATTGTTTT -3';

ERK1/2 124 bps $\{$ F $\} 5^{\prime}$-CCTGAACATGACCACACTGG-3 ${ }^{\prime}$

$\{\mathrm{R}\} 5^{\prime}$-TGCATCCCACAGACCAAATA-3';

JNK 176 bps $\{$ F $\} 5^{\prime}$-CAAACCTCTGCCAGGTCATT-3'

$\{\mathrm{R}\} 5^{\prime}$-GCCGAAATCCAAAATCTTCA-3';

IкBa 184 bps\{F\} 5'-TTTCGGAGGAGATGGAGAGA-3'

$\{\mathrm{R}\} 5^{\prime}$-CTGTTCAGGTACGGGTCGTT-3'; and

NF-кB 154 bps $\{$ F $\} 5^{\prime}$-GAGCCCTTTGTGCAAGAGAC-3'

\{R\} 5'-TGGGATACGTCCTCCTGTTC- ${ }^{\prime}$

The thermocycling conditions were initial denaturation at $95{ }^{\circ} \mathrm{C}$ for $10 \mathrm{~min}$, followed by $95{ }^{\circ} \mathrm{C}$ for 10 $\mathrm{s}$ and $60{ }^{\circ} \mathrm{C}$ for $30 \mathrm{~s}$. All reactions were performed in triplicate for 40 cycles using a Stratagene Mx3005P system. Relative quantitation was performed using standard curves for each primer (sequence information). The amount of mRNA was normalized to that of $\beta$-actin.

\section{Statistical analysis}

The data are presented as the mean \pm standard deviation (SD). Survival analysis was evaluated with the Kaplan-Meier method. Comparisons between curves were made with the log-rank test. Mean values were analysed by multivariate analysis of variance. Comparisons between groups were made by the LSD test. All data were analysed with the Statistical Package for the Social Sciences (SPSS; version 20.0). P-values $<0.05$ were considered statistically significant.

\section{Results}

Effects of PHN on the survival of zebrafish challenged with lethal LPS

To test whether PHN could protect zebrafish from a lethal challenge with LPS, the mortality rates of zebrafish treated with different doses of PHN were analysed. All of the zebrafish challenged with $0.5 \mathrm{mg} / \mathrm{ml}$ LPS died within $48 \mathrm{hpi}$ (Fig. 2). In contrast, the zebrafish treated with PHN at concentrations of $1,3.3$, and $10 \mu \mathrm{g} / \mathrm{ml}$ died later, and a dose-dependent increase in survival was observed. All the zebrafish survival rates in the PHN treatment groups were greater than $24 \%$ within $48 \mathrm{hpi}$. Treatment with $1 \mu \mathrm{g} / \mathrm{ml}$ PHN did not increase zebrafish survival but did delay time of death $(\mathrm{P}<0.0001)$, as $26.1 \%$ of the zebrafish survived through $48 \mathrm{hpi}$, while no zebrafish were alive at $96 \mathrm{hpi}$. However, $3.3 \mu \mathrm{g} / \mathrm{ml} \mathrm{PHN}$ not only delayed the zebrafish time of death but also decreased the number of deaths $(\mathrm{P}<0.0001)$; the survival rate increased to $17 \%$ at $96 \mathrm{hpi}$. The zebrafish administered $10 \mu \mathrm{g} / \mathrm{ml} \mathrm{PHN}$ or $5 \mu \mathrm{g} /$ ml DEX exhibited significantly increased survival, with 65\% and 53\% survival observed at 48 hpi, respectively. By 96 hpi, their survival rates were still greater than 30\%. Meanwhile, 


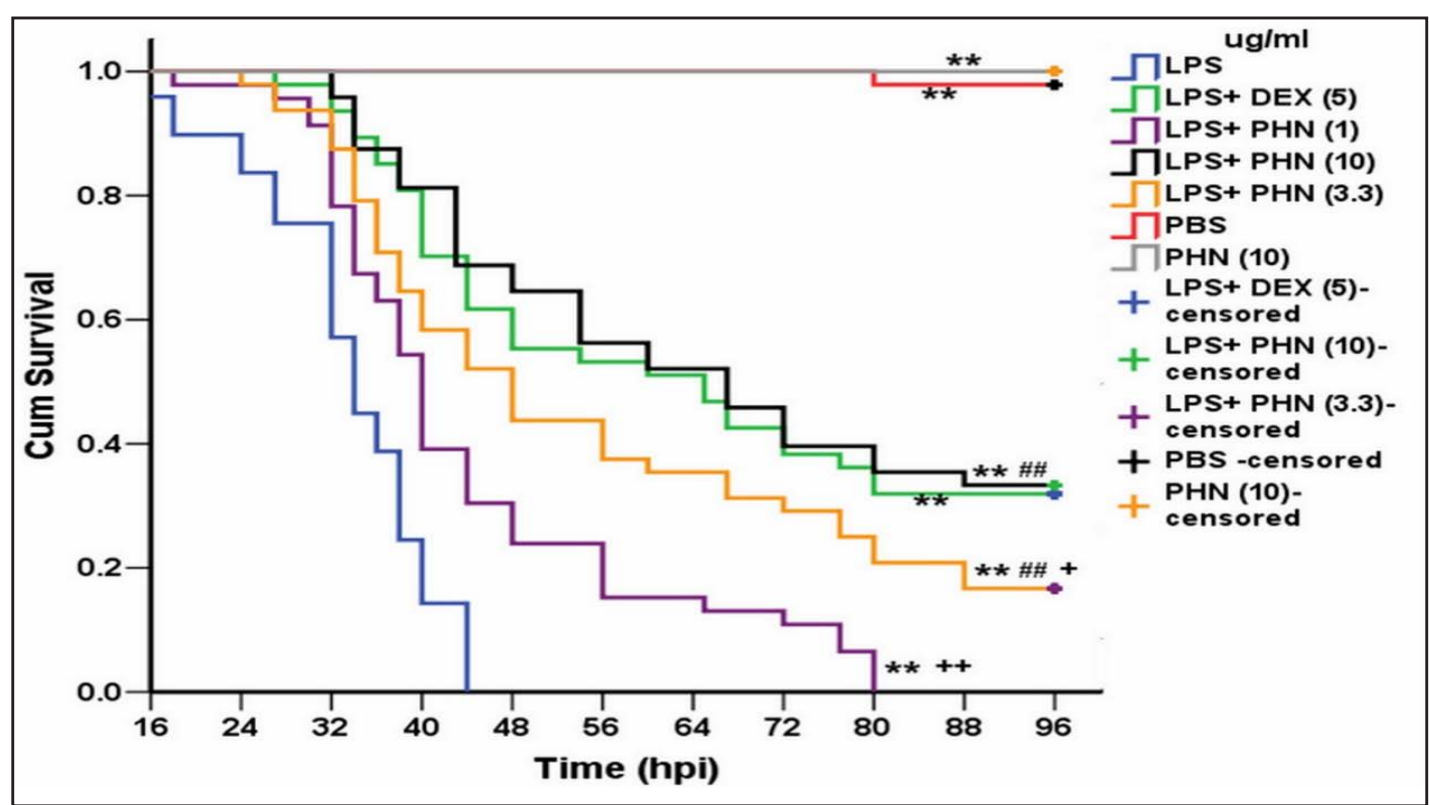

Fig. 2. Protective effects of PHN and DEX on zebrafish challenged by LPS. Three hundred and fifty larvae were randomly divided into 7 groups $(n=50)$. Survival of zebrafish larvae exposed to the indicated doses of PHN after a $0.5 \mathrm{mg} / \mathrm{ml}$ injection of LPS into their yolks at $3 \mathrm{dpf}$ was assessed. The negative and positive controls were PBS and $5 \mu \mathrm{g} / \mathrm{ml}$ DEX, respectively. The mortalities of the zebrafish were observed for $96 \mathrm{~h}$ after administration. ** $\mathrm{P}<0.001$ relative to the LPS group; \#\# $\mathrm{P}<0.001$ between the $1 \mu \mathrm{g} / \mathrm{ml} \mathrm{PHN}$ treatment group and the 10 and $3.3 \mu \mathrm{g} / \mathrm{ml}$ PHN treatment groups; $+\mathrm{P}<0.05$ between the 10 and $3.3 \mu \mathrm{g} / \mathrm{ml}$ PHN treatment groups. Each curve represents data pooled from three independent experiments.

the median survival time among the PHN concentrations $(1,3.3$, and $10 \mu \mathrm{g} / \mathrm{ml})$ were $40 \pm$ $1.2,48 \pm 3.9$, and $67 \pm 7.8 \mathrm{~h}$, respectively; LPS and $5 \mu \mathrm{g} / \mathrm{ml}$ DEX were $34 \pm 1.2$ and $65 \pm 10.8$ $\mathrm{h}$, respectively. PHN could markedly extend the median survival time of zebrafish, and the protection provided by $10 \mu \mathrm{g} / \mathrm{ml}$ PHN was much better than that provided by 1 and $3.3 \mu \mathrm{g} /$ $\mathrm{ml}$ PHN $(\mathrm{P}<0.05)$. The above results confirmed that suitable doses of PHN could provide significant protection to zebrafish challenged with lethal LPS. We used $10 \mu \mathrm{g} / \mathrm{ml}$ PHN as our immersion concentration and focused on inflammation grading, neutrophil activation, pro-inflammatory cytokine expression and the inflammatory signalling pathway in zebrafish larvae at different time points after treatment with PHN.

Effects of PHN on inflammation grading in zebrafish yolks in vivo

To determine the effects of PHN on LPS-mediated neutrophil recruitment in inflammatory response in vivo, inflammation grading was evaluated at 12 and 24 hpi using a Tg (mpo:GFP) line. At $3.5 \mathrm{dpf}$, neutrophils mainly appeared in the PBI, and both the head stroma and epidermis expressed only a few of these cells [32]. Yolks with severe neutrophil infiltration were observed $12 \mathrm{~h}$ after being injected with LPS (Fig. 3-B), but only a small number of sparsely distributed neutrophils were observed in PHN-treated larvae (Fig. 3-D). The PBS negative control and DEX-treated group produced similar results (Fig. 3-A, C). At 24 hpi, tracking neutrophils in vivo showed severe yolk deformation, necrosis, and only a few neutrophils remaining in the LPS group (Fig. 3-B'). In contrast, the zebrafish administered PHN exhibited only some neutrophil recruitment in their yolks, and no necrosis was observed (Fig. 3-D', C'). Meanwhile, treatment with PHN markedly decreased the inflammation grading of larvae compared with that of the LPS group at the abovementioned time points (Fig. 3-E, $\mathrm{F}, \mathrm{n}=20, \mathrm{P}<0.001$ ). No neutrophils or necrosis were found in the zebrafish yolks of the PBS group at 24 hpi (Fig. 3-A', F). These results showed that PHN could significantly reduce the infiltration of neutrophils and decrease the extent of necrosis induced by LPS. 

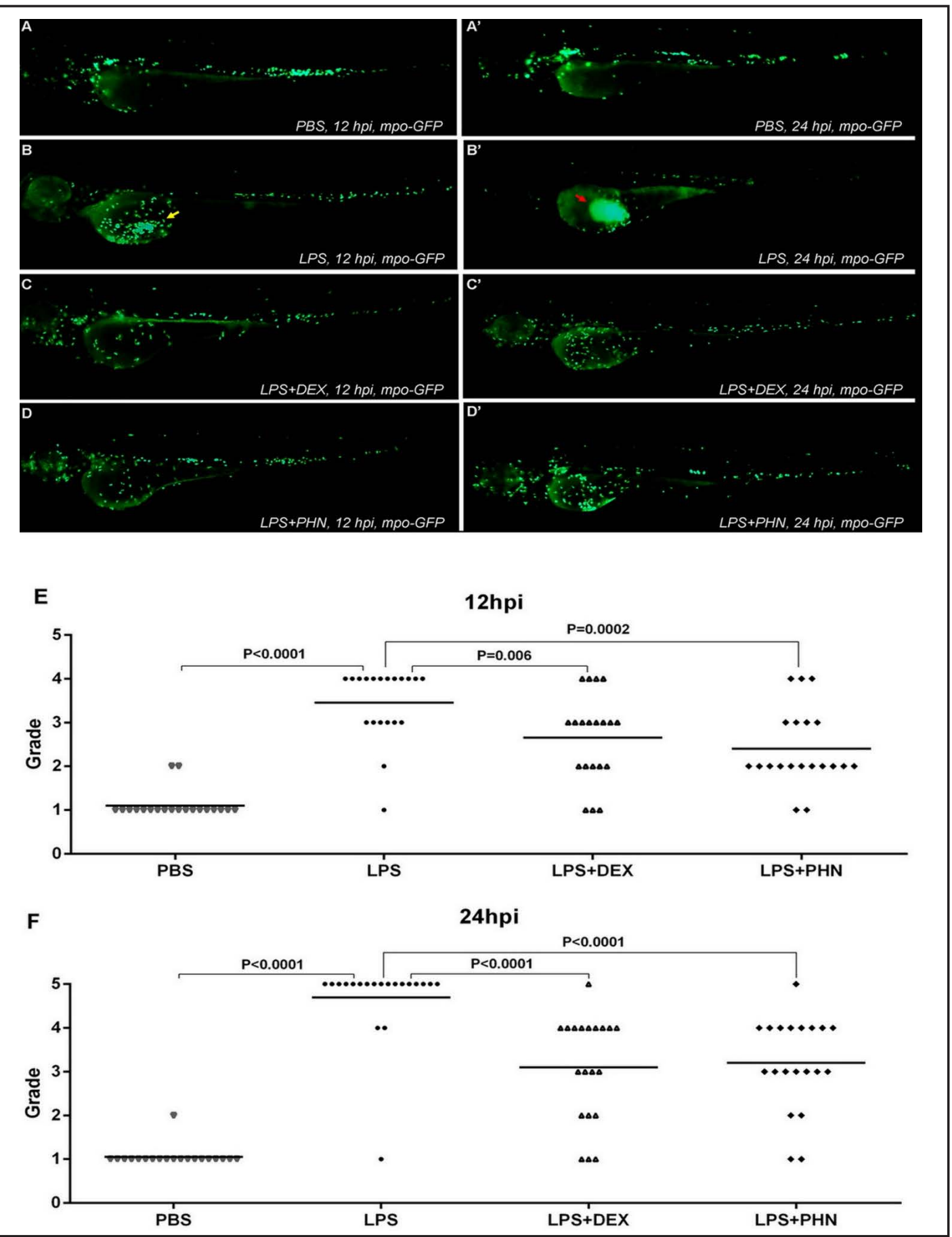

Fig. 3. PHN inhibits neutrophil recruitment and decreases the yolk necrosis induced by LPS; Tg (mpo:GFP) marks neutrophils (A-D'). Fluorescence imaging of 3-dpf Tg (mpo:GFP) live larvae $(\mathrm{n}=20)$ at 12 hpi (A, B, C, D) and 24 hpi (A', B', C', D') using the GFP channel. The yellow arrow in B indicates the neutrophil, while the red arrow in $\mathrm{B}^{\prime}$ indicates yolk necrosis. The negative control was PBS (A/A'), and the positive control was 5 $\mu \mathrm{g} / \mathrm{ml}$ DEX (C/C'). The LPS model group (B/B') and the $10 \mu \mathrm{g} / \mathrm{ml}$ PHN treatment group (D/D') are shown. E and $\mathrm{F}$ show the inflammatory grading scatter diagrams in each group at 12 and $24 \mathrm{hpi}$, respectively. Grade 1 = normal (zero or several leucocytes, no necrosis); Grade 2 = leucocytes scattered in the yolk, no necrosis; Grade 3 = leucocyte recruitment to the LPS site, no necrosis; Grade 4 = bulk infiltration of leucocytes and leucocyte adhesion, no necrosis; Grade 5 = yolk deformation and necrosis. The scatter diagrams represent data pooled from three independent experiments. 


\section{Effects of PHN on neutrophil production and migration}

Previous studies have demonstrated that SB can be used to detect the presence of neutrophils with dark colour [32,33]. Here, to quantify the activation or migration of neutrophils and to determine the significance of the treatment group, migration quantity and directionality were calculated from the tracks of $\mathrm{SB}^{+}$cells (Fig. 4-A, red arrow) migrating towards or away from the injection point. As expected, an intense increase in the total number of $\mathrm{SB}^{+}$cells in the LPS group compared with that in the PBS group was observed from 1 to 12 $\mathrm{h}$ (Fig. 4-B, $\mathrm{n}=10, \mathrm{P}<0.0001$ ). However, at $24 \mathrm{hpi}$, the total number of $\mathrm{SB}^{+}$cells was sharply decreased, with obvious deformation and necrosis in the yolks observed (smaller than that of the PBS group, $\mathrm{P}<0.0001$ ). The total number of $\mathrm{SB}^{+}$cells in the PHN-treated group was obviously less than that in the LPS group from 1 to $12 \mathrm{~h}$, which was similar to that in the PBS group. In the yolk sacs, $\mathrm{SB}^{+}$cells (Fig. 4-C) in the LPS group accumulated with time, as they peaked at 6 hpi (NO. $=179.7 \pm 11.7)$ and then declined. A sharp decrease in the number of $\mathrm{SB}^{+}$cells at the injection site was observed by $24 \mathrm{hpi}(\mathrm{NO} .=30.4 \pm 1.7)$. In the AOs (Fig. 4-D), $\mathrm{SB}^{+}$cells in the LPS group continuously decreased for up to $24 \mathrm{hpi}(\mathrm{NO} .=39.1 \pm 2.8$ ) after peaking at $3 \mathrm{hpi}(\mathrm{NO} .=104.6 \pm 6.4)$. In contrast, the number of cells in the yolks peaked at $6 \mathrm{hpi}$, but few $\mathrm{SB}^{+}$cells remained at 12 and $24 \mathrm{hpi}$ in the PBS group (Fig. 4-C). The number of cells in the AOs consecutively increased after $6 \mathrm{~h}$ (Fig. 4-D). Notably, treatment with PHN remarkably decreased the number of yolk cells at the corresponding time points, except at $24 \mathrm{hpi}$. While the $\mathrm{SB}^{+}$cell counts in the PHN group was greater than that in the LPS group at $24 \mathrm{hpi}$, necrosis was not observed (Fig. 4-C, $\mathrm{P}<0.0001$ ). Meanwhile, the number of $\mathrm{SB}^{+}$cells in the AOs declined consecutively after peaking at $3 \mathrm{hpi}$. Similar results were observed in the DEX group. The above results demonstrated that PHN could markedly diminish neutrophil production and inhibit neutrophil migration and activation from $1 \mathrm{~h}$ to $24 \mathrm{~h}$ when stimulated by LPS.

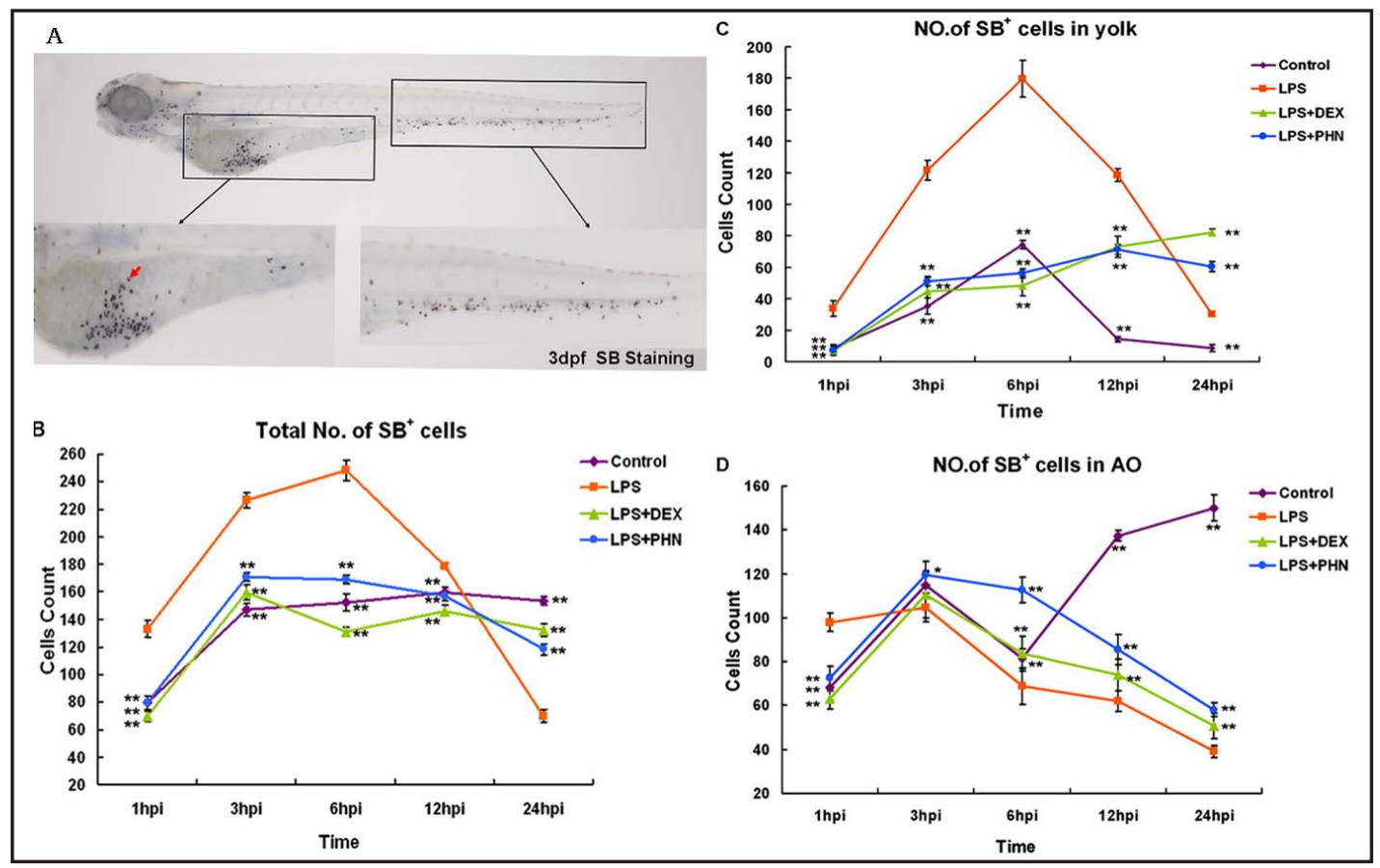

Fig. 4. PHN inhibits neutrophil production and migration. (A) SB staining to label neutrophils at 3 dpf. The red arrow shows the neutrophil $(\mathrm{n}=10)$. (B) Linear chart revealing the total number of $\mathrm{SB}^{+}$cells in zebrafish from 1 to $24 \mathrm{~h}$ after treatment with $5 \mu \mathrm{g} / \mathrm{ml}$ DEX and $10 \mu \mathrm{g} / \mathrm{ml} \mathrm{PHN} ; * * \mathrm{P}<0.001$ compared to the LPS group. (C) Linear chart revealing the number of $\mathrm{SB}^{+}$cells in the yolk sac from 1 to $24 \mathrm{~h}$ after treatment with DEX and PHN; * P<0.001 compared to the LPS group. (D) Linear chart revealing the number of neutrophils in the AOs from 1 to $24 \mathrm{~h}$ after treatment with DEX and PHN. Negative controls were performed using PBS; $*$ P $<0.05$ and $* * \mathrm{P}<0.001$ compared to the LPS group. 


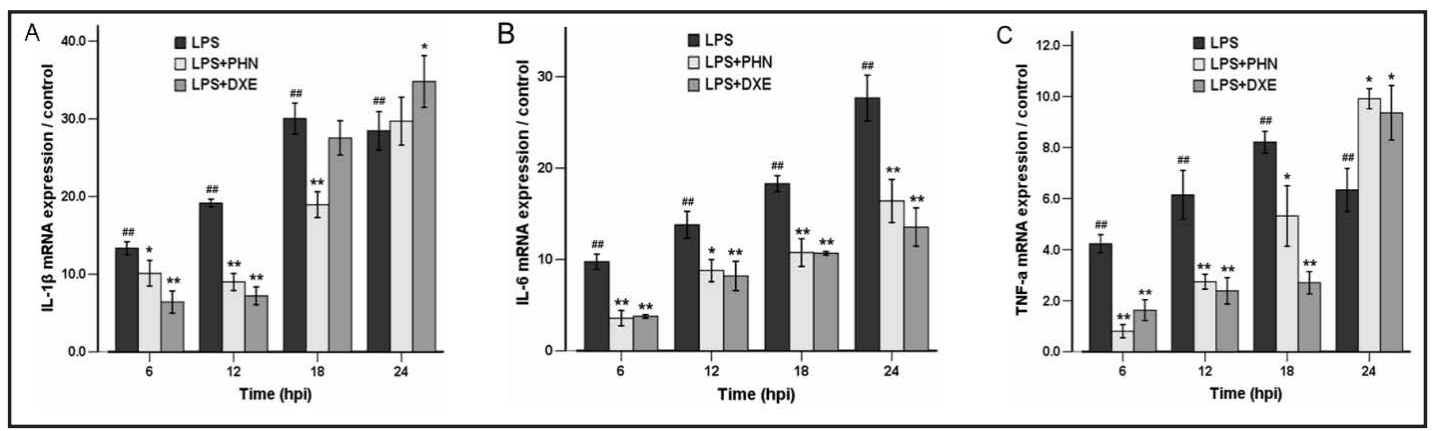

Fig. 5. PHN reduces the expression of the proinflammatory cytokines TNF- $\alpha$, IL-1 $\beta$, IL-6. Zebrafish larvae injected with $0.5 \mathrm{mg} / \mathrm{ml}$ LPS at $3 \mathrm{dpf}$ were sampled at $6,12,18$, and 24 hpi to measure TNF- $\alpha$, IL- 6 and IL-1 $\beta$ mRNA using RQ-PCR (A-C). (A) Group comparison of IL-1 $\beta$ expression the same time; $* \mathrm{P}<0.05$ and $* *$ $\mathrm{P}<0.001$ compared to the LPS group. (B) Group comparison of IL-6 expression at the same time; $* \mathrm{P}<0.05$ and $* * \mathrm{P}<0.001$ compared to the LPS group. (C) Group comparison of TNF- $\alpha$ expression at the same time; $* \mathrm{P}<0.05$ and $* * \mathrm{P}<0.001$ compared to the LPS group. \# $\mathrm{P}<0.05$ and \#\# $\mathrm{P}<0.001$ compared to the control group.

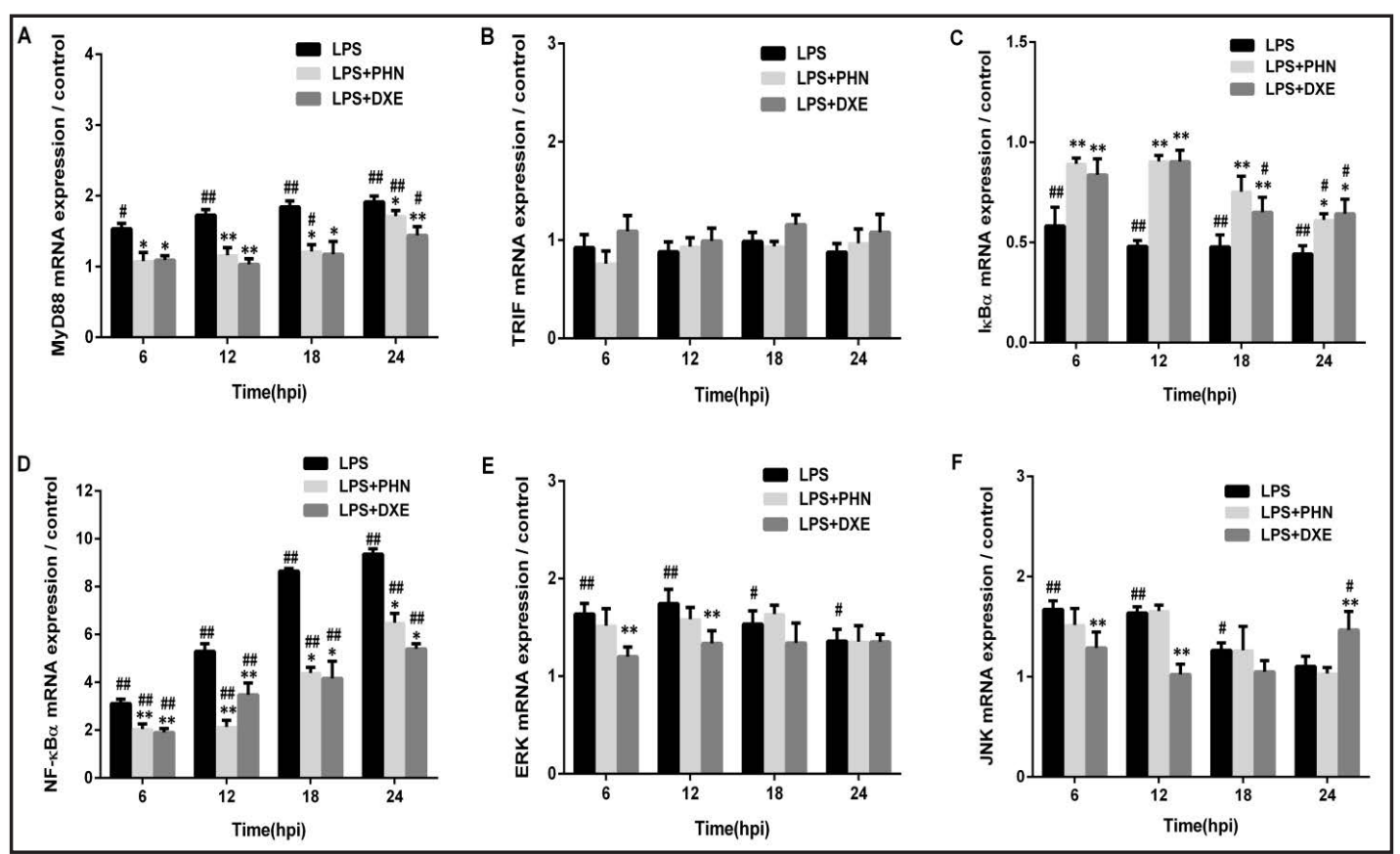

Fig. 6. PHN inhibits LPS-induced inflammation by suppressing MyD88/NF- $\kappa B$ signalling. Zebrafish larvae injected with $0.5 \mathrm{mg} / \mathrm{ml}$ LPS at $3 \mathrm{dpf}$ were sampled at 6, 12, 18, and 24 hpi to measure MyD88, TRIF, IкB $\alpha$,

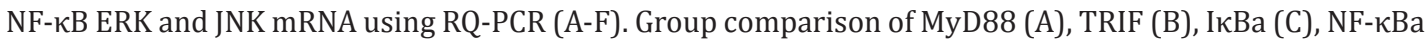
(D), ERK (E) and JNK (F) expression at the same time; $* \mathrm{P}<0.05$ and $* * \mathrm{P}<0.001$ compared to the LPS group; \# $\mathrm{P}<0.05$ and \#\# $\mathrm{P}<0.001$ compared to the control group.

Effects of PHN on the mRNA expression of $I L-1 \beta, I L-6$, and TNF- $\alpha$

Because LPS-treated leukocytes can produce the pro-inflammatory cytokines IL-1 $\beta$, IL-6 and TNF- $\alpha$, which contribute to inflammation $[11,34]$, we detected the expression of these cytokines. As shown in Fig. 5, IL-1 $\beta$, IL-6, and TNF- $\alpha$ expression was markedly increased in LPS-infected larvae from 6 to 24 hpi compared with PBS-injected larvae, but the PBSinjected larvae expression levels and changes were different at these time points (Fig. 5). IL-1 $\beta$ showed an immediate response to LPS infection, as its expression markedly increased 


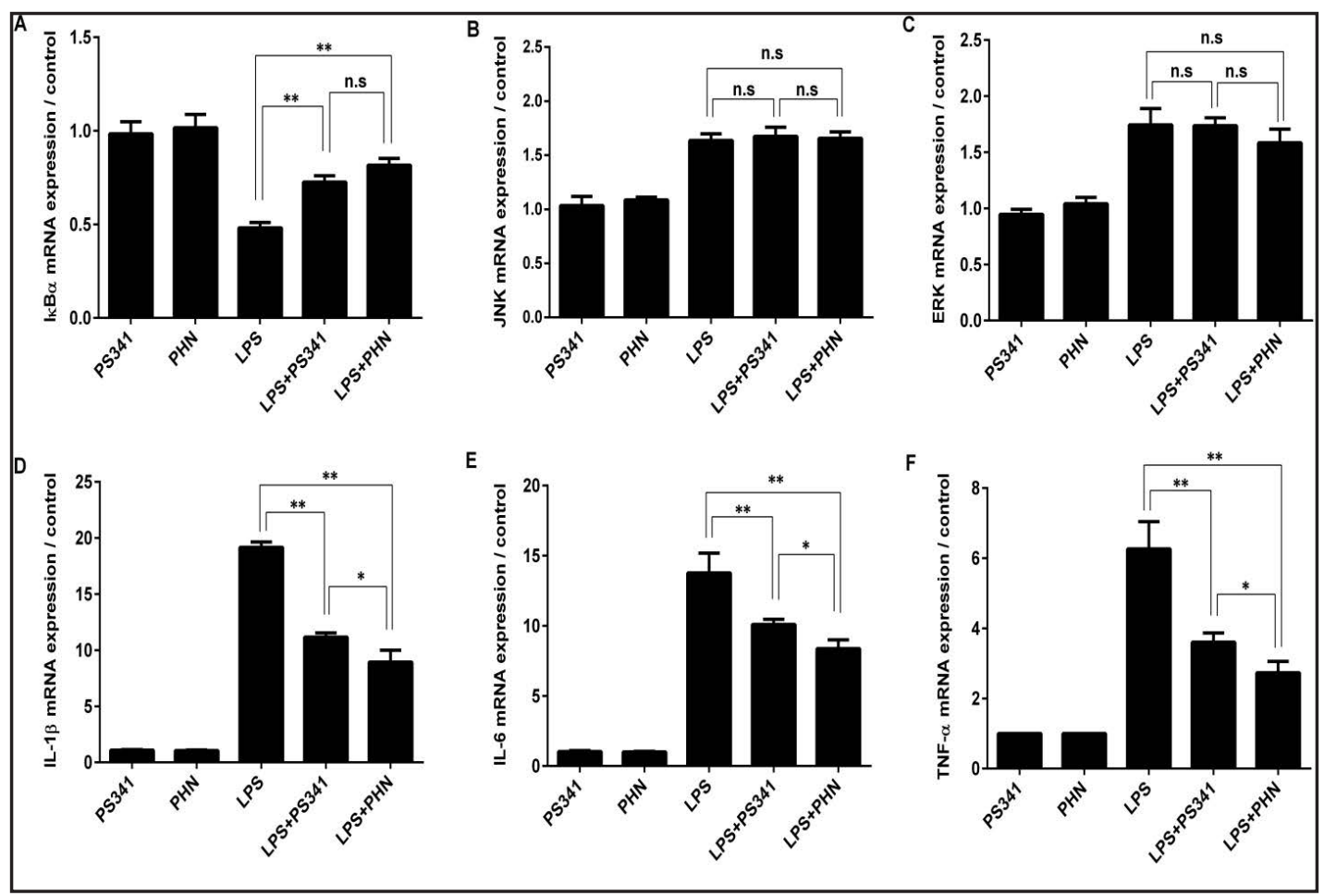

Fig. 7. The inhibitory effects of PHN and SP341 on the LPS-activated MyD88/NF- $\kappa$ B pathway. Zebrafish were pretreated for $12 \mathrm{~h}$ with PHN or PS341 after they were injected with LPS for $0.5 \mathrm{~h}$. The mRNA expression levels of IkBa (A), JNK (B), ERK1/2 (C), IL-1 $\beta$ (D), IL-6 (E) and TNF- $\alpha$ (F) were then determined; * P<0.05 and $* * \mathrm{P}<0.001$ compared between the indicated groups; n.s indicates no statistically significant difference.

(30-fold) at 18 hpi. PHN could significantly reduce the IL-1 $\beta$ expression up-regulated by LPS from 6 to 18 hpi (Fig. 5-A, P<0.05) but could not decrease IL-1 $\beta$ expression at 24 hpi. After LPS stimulation, IL- 6 was remarkably expressed in zebrafish in a time-dependent manner from 6 to $24 \mathrm{hpi}$ and was inhibited in the presence of PHN (Fig. 5-B, P<0.05). Meanwhile, the up-regulated TNF- $\alpha$ expression levels (Fig. 5-C) were smaller than those of IL-1 $\beta$ and IL- 6 at each corresponding time point. Similar to IL-1 $\beta$, TNF- $\alpha$ expression up-regulated by LPS was significantly suppressed by PHN before $18 \mathrm{hpi}(\mathrm{P}<0.001)$ but was not supressed at $24 \mathrm{hpi}$ $(\mathrm{P}>0.05)$. The same results were observed in the DEX treatment group $(\mathrm{P}<0.05)$. Although the up-regulated levels of IL-1 $\beta$, IL- 6 and TNF- $\alpha$ varied from 6 to $18 \mathrm{~h}$, they showed the same PHN inhibition tendency during LPS-induced inflammation.

\section{PHN inhibits LPS-induced inflammation by suppressing MyD88/NF- $\kappa B$ signalling}

TLR signalling is divided into two distinct signalling pathways in mammals, the MyD88dependent and the TRIF-dependent pathway. The MyD88-dependent pathway leads to the expression of pro-inflammatory genes, such as TNF, IL-1 $\beta$, IL-6, through the activation of NF$\kappa \mathrm{B}$, while the TRIF-dependent pathway does not. Therefore, we hypothesized that the antiinflammatory effects of PHN are potentially associated with the MyD88-dependent pathway in zebrafish. To confirm this hypothesis, we observed MyD88 and TRIF expression. Our results showed that stimulating zebrafish with LPS resulted in increased MyD88 expression from 6 to $24 \mathrm{hpi}$, which was obviously prevented by PHN (Fig. 6A, P<0.05). By contrast, LPS and PHN did not affect the expression of TRIF (Fig. 6B, P>0.05).

Since MyD88 has been found to activate NF- $\mathrm{KB}$, a critical transcriptional regulator for inflammatory-related gene coding in response to LPS [35], we examined the effects of PHN

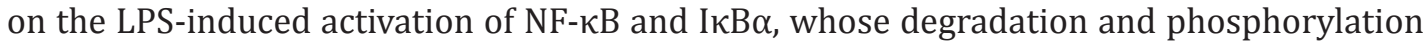
result in the release and transfer of NF- $\kappa B$ into the nucleus [36]. As shown in Fig. 6C, after LPS 
stimulation, the mRNA IкB $\alpha$ expression levels were remarkably decreased from 6 to $24 \mathrm{hpi}$ in zebrafish $(\mathrm{P}<0.05)$, while NF- $\kappa \mathrm{B}$ expression significantly increased in a time-dependent manner (Fig. 6D, P<0.05). However, PHN treatment prevented this phenomenon (Fig. 6CD). These results suggest that the anti-inflammatory effect of PHN is associated with the MyD88/I $\kappa \alpha / N F-\kappa B$ signalling pathway.

Recently, some studies have indicated that the LPS-induced production of inflammatory cytokines is strongly affected by MAPKs, which include ERK1/2 and JNK [37-39]. We thus determined the effects of PHN on the LPS-induced activation of MAPKs in zebrafish. We found that the expression levels of ERK1/2 (Fig. 6E) and JNK (Fig. 6F) were up-regulated by LPS $(\mathrm{P}<0.05)$; however, PHN did not affect this up-regulation from 6 to 24 hpi (Fig. 6E-F, $\mathrm{P}>0.05)$. These findings demonstrate that while PHN attenuates LPS-induced inflammation in zebrafish, this effect is likely not associated with the ERK1/2 and JNK signalling pathways.

Comparison of the inhibitory effects of PHN and signalling inhibitors on the LPS-activated MyD88/NF- $\kappa$ B pathway

Since we observed that PHN suppressed the LPS-triggered activation of NF- $\kappa B$, we next determined the relative contribution of the anti-inflammatory activity of PHN to inhibiting these signalling pathways. We used the pharmaceutical inhibitor PS341 to specifically block IкBa degradation. Zebrafish were pretreated for $12 \mathrm{~h}$ with PHN or PS341 after they were injected with LPS for $0.5 \mathrm{~h}$. The mRNA expression levels of I $\mathrm{B} \alpha \alpha, \mathrm{JNK}$, ERK1/2, IL$1 \beta$, IL- 6 and TNF- $\alpha$ were then determined. As shown in Fig. 7A-C, PS341 inhibited only the phosphorylation of IkBa and increased the expression of IkBa, demonstrating the specificity of the inhibitor we used in our experiments. Regarding IL-1 $\beta$, IL- 6 and TNF-a production, PS341 significantly reduced the LPS-induced up-regulation of these cytokines compared to that of the LPS-stimulated group. However, when compared to the PHN-treated group, PS341 could not exhibit the same inhibition (Fig. 7D-F, $\mathrm{P}<0.05$ ).

Taken together, these findings demonstrate that PHN inhibits LPS-induced lethal neutrophil inflammation in zebrafish by suppressing the MyD88/I $\mathrm{B} \alpha / \mathrm{NF}-\kappa \mathrm{B}$ pathway.

\section{Discussion}

Cells or rodents are traditionally used to study and screen antiendotoxic drugs [40-42]. However, drugs that have antiendotoxic effects in vitro may be ineffective in vivo. Conversely, drugs that are effective only in vivo can sometimes be unidentifiable with in vitro screens. Rodent screening models also have many shortcomings, as they are time consuming, strenuous and costly. Due to unique advantages in biology, genomics, and genetics, as well as high conservativity in the disease signal transduction pathway, drug screens in zebrafish embryos and larvae hold considerable promise in filling the gap between cell and rodent models $[16,34,43]$. In the present study, we show a simple, quick and practical method for screening and evaluating antiendotoxic agents using zebrafish embryos. Previous studies found that PHN could depress inflammation in vitro (28). Herein, we showed that PHN suppressed LPS-stimulated inflammation in vivo.

Kaplan-Meier survival curves are usually used to represent the efficacy of new antiendotoxic drugs. Cumulative survival from a curve is expressed with the drug stimulus or action time. The detection of different drug challenge doses on equivalent doses of LPS, as well as the formation of actual dose-response curves, provides valuable statistics for the antiendotoxic drug being studied. Thus, the clinical efficacy of the experimental therapy can be tested [44]. Here, utilization of zebrafish cumulative survival as a hard, primary end-point for evaluating the efficacy of PHN was very useful. We analysed the survival of zebrafish treated with different doses of PHN for 96 hpi. The data presented in Fig. 2 indicate that PHN could prolong the survival time of zebrafish and increased their survival rate in a dose-dependent manner compared to the LPS group. These results could provide essential information for evaluating PHN efficacy in a pre-clinical trial. 


\section{Cellular Physiology Cell Physiol Biochem 2017;43:2074-2087 \begin{tabular}{l|l|l}
\hline and Biochemistry 10.1159/000484192 & $\begin{array}{l}\text { C) } 2017 \text { The Author(s). Published by S. Karger AG, Basel } \\
\text { www.karger.com/cpb }\end{array}$ \\
\hline
\end{tabular}

Neutrophils play pivotal roles in the LPS stimulation of inflammatory diseases. During early periods of inflammation, neutrophils circulating in the blood are the first and most abundant leukocytes to arrive at the inflammation point to eliminate the causative organism and to resist infection [45]. However, overactive neutrophils have been shown to aggravate inflammatory tissue injury, while reduced neutrophil excessive exudation was shown to attenuate inflammation [46-48]. Some reports have also shown that neutrophil recruitment is markedly defective in the advanced stages of severe infectious diseases, and patients with low neutrophil levels (high neutrophil necrosis) have an increased risk for these diseases [4951]. Here, decreased neutrophil (MPO:GFP/ $/ \mathrm{SB}^{+}$cells) recruitment to the yolk (injection point) and a decreased total number of neutrophils were observed in the PHN-treated group before $12 \mathrm{hpi}$. Moreover, a sharp reduction in the total number of neutrophils and obvious necrosis was observed at $24 \mathrm{hpi}$, while PHN treatment prevented this phenomenon. This result was similar to previous research [52] reporting that PHN could enhance immunological functions in mice. Neutrophil necrosis and apoptosis are known to be related to immunoregulation function. The PHN-dependent enhancement of immunological function might significantly inhibit the sharp reduction of neutrophils in the advanced stages of inflammatory diseases.

In response to the LPS stimulus, a series of intracellular signalling cascades are initiated, which ultimately lead to the activation of neutrophils and their release of pro-inflammatory cytokines. MyD88, the first responsive intracellular signalling molecule, has been implicated as a key modulator in the inflammatory signalling cascades triggered by LPS, including the NF- $\kappa B$ and MARK pathways [8]. As shown in our results, PHN obviously inhibited the LPSinduced expression of MyD88, but not of TRIF, indicating that PHN could suppress LPSinduced inflammation in the MyD88-dependent pathway.

The nuclear transcription factor NF- $\kappa \mathrm{B}$ is a potential positive regulator that stimulates the release of pro-inflammatory cytokines during inflammation [53]. Our present data indicated that PHN suppressed the degradation of I $\mathrm{B} \alpha$ and the transactivational activity of NF- $\kappa \mathrm{B}$ in LPS-stimulated zebrafish models to extents similar to that of the proteasome inhibitor PS341. We also observed that PHN apparently attenuated the LPS-triggered expression of TNF- $\alpha$, IL- 6 and IL-1, suggesting that PHN inhibits LPS-induced lethal inflammation in vivo by suppressing the MyD88/I $\kappa \mathrm{B} \alpha / \mathrm{NF}-\kappa \mathrm{B}$ signalling pathway.

The MARK signalling pathway plays pivotal roles in immune and inflammatory responses. In particular, some evidence has shown that the ERK1/2 MAPK and JNK MAPK signalling pathways are related to leukocyte migration and pro-inflammatory cytokine expression [10, 37]. Previous studies revealed that the inhibition of ERK, as well as the inhibition of proinflammatory cytokines and chemokines, using specific inhibitors effectively attenuated acute lung injury by reducing lung neutrophilia and hyperpermeability [54]. PHN also significantly inhibited ERK and p38 pathway activation in acute lung injury [55]. However,

Fig. 8. The speculated anti-inflammatory network of PHN. $\perp$ indicates an inhibitory effect. PHN exerts antiinflammatory effects and suppresses the expression of MyD88, IkBa and NF- $\kappa$ B, as well as the release of inflammatory factors induced by LPS, by suppressing the MyD88-dependent inflammatory pathway in vivo.

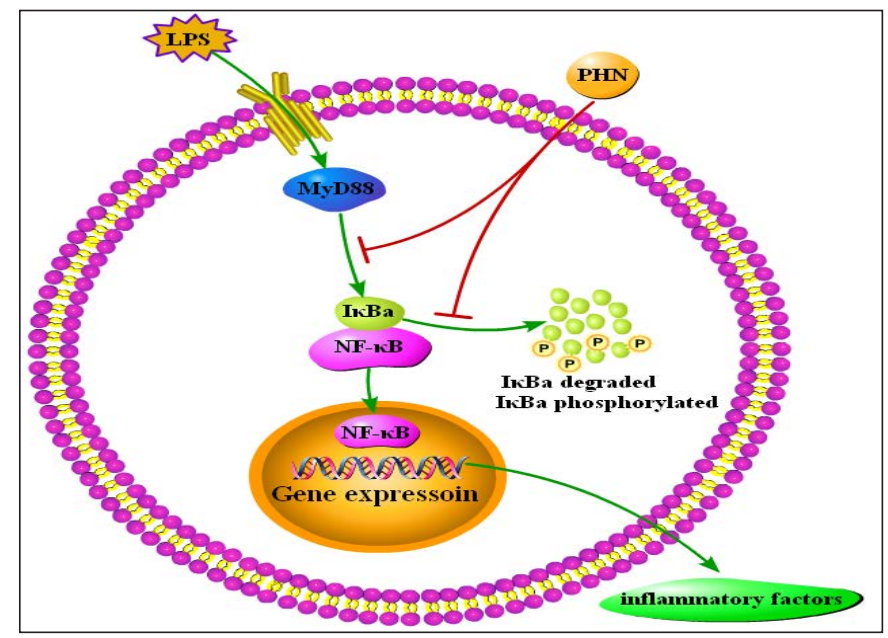


in this study, we determined only the effects of PHN on the expression of JNK and ERK1/2 to show that no statistically significant differences existed in JNK and ERK1/2 activation. Further investigating the effects of PHN on the activity of MAPK subunits, such as p38 MAPK, in the future will be worthwhile.

In summary, we first found that PHN exhibits significant anti-inflammatory effects against zebrafish stimulated with LPS by reducing neutrophil infiltration, attenuating necrosis and inhibiting proinflammatory cytokine expression. We elucidated that the underlying mechanism involves the suppression of MyD88-NF- $\kappa \mathrm{B}$ signalling (Fig. 8). Our study provides a rationale for the clinical application of PHN as an anti-inflammatory agent.

\section{Disclosure Statement}

The authors declare no conflicts of interest.

\section{Acknowledgements}

This study was supported by grants from the National Natural Science Foundation of China (No.81573671), the Natural Science Foundation of Guangdong Province, China(No.2014A03031) and the Administration of Traditional Chinese Medicine of Guangdong Province, China (No.20171274). The authors thank the staff of the Key Laboratory of Zebrafish Modeling and Drug Screening for Human Diseases Institute at Southern Medical University for their expert care of the zebrafish and their guidance and help provided in this study.

\section{References}

1 Guha M, Mackman N: LPS induction of gene expression in human monocytes. Cell Signal 2001;13:85-94.

-2 Beutler B, Rietschel ET: Innate immune sensing and its roots: The story of endotoxin. Nat Rev Immunol 2003;3:169-176.

3 Kasper DL, Harrison TR: Harrison's principles of internal medicine. McGraw-Hill Medical Pub, New York 2005; Division 60.

4 Haslett C: Resolution of inflammation and the role of apoptosis in the tissue fate of granulocytes. Clin Sci 1992;83:639-648.

5 Mcleish K, Klein J, Coxon P, Head KZ, Ward RA: Bacterial phagoeytosis activates extxacellular signalregulated kinase and p38 mitogen-activated protein kinase caseacles in human neutrophils. J Leukoc Biol 1998;64:835-844.

6 Sweeney T, Nguyen P, Omann G, Hinshaw DB: Lipopolysaccharide protects polymorphonuclear leukoeytes from apoptosis via tyrosine phosphoryl ation-dependent signal transduction pathways. J Surgl Res 1998;74:64-70.

7 Takeda K, Akira S: Toll-like receptors in innate immunity. Int Immunol 2005;17:1-14

8 O'Neill LA, Bowie AG: The family of five:TIR-domain-containing adaptors in Toll-like receptor signalling. Nat Rev Immunol 2007; 7:353-364.

-9 Yamamoto M, Sato S, Hemmi H, Hoshino K, Kaisho T, Sanjo H, Takeuchi O, Sugiyama M, Okabe M, Takeda K, Akira S: Role of adaptor TRIF in the MyD88-independent toll-like receptor signaling pathway. Science 2003;301:640-643.

10 Bruggen T, Nijenhuis S, van Raaij E, Verhoef J, van Asbeck BS: Lipopolysaccharide-induced tumor necrosis factor alpha production by human monocytes involves the raf-1/MEK1-MEK2/ERK-ERK2 pathway. Infect Immun 1999;67:3842-3829.

11 Hatanaka E, Shimomi FM, Curi R: Sodium chloride inhibits cytokine production by LPS-stimulated human neutrophils and mononuclear. Shock 2007;27:32-35.

12 Lesley R, Ramakrishnan L: Insights into early mycobacterial pathogenesis from the zebrafish. Curr Opin Microbiol 2008;11:277-283. 


\section{Cellular Physiology Cell Physiol Biochem 2017;43:2074-2087 \begin{tabular}{l|l|l}
\hline DOI: 10.1159/000484192 2017 The Author(s). Published by S. Karger AG, Basel & (c)
\end{tabular} and Biochemistry Published onlıne: October 23, 2017 www.karger.com/cpb}

Yang et al.: Protective Effect of PHN in Zebrafish

13 Lieschke GJ, Trede NS: Fish immunology. Curr Biol 2009;19:678-682.

14 Traver D, Herbome P, Patton EE: The zebrafish as a model organism to study development of the immune system. Adv Immunol 2003;81:253-330.

15 Trede NS, Langenau DM, Traver D, Look AT, Zon LI: The use of zebrafish to understand immunity. Immunity 2004;20:367-379.

16 Annemarie HM, Spaink HP: Host-Pathogen Interactions Made Transparent with the Zebrafish Model. Curr Drug Targets 2011;12:1000-1017.

17 Renshaw SA, Loynes CA, Trushell DM, Elworthy S, Ingham PW, Whyte MK: A transgenic zebrafish model of neutrophilic inflammation. Blood 2006;108:3976-3978.

18 National Commission of Chinese Pharmacopoeia, 2015. Pharmacopoeia of Peoples Republic of China, Chemical Industry Press, Beijing, 1997, pp 170-171.

19 Ko HC, Wei BL, Chiou WF: Dual regulatory effect of plant extracts of Forsythia suspense on RANTES and MCP-1 secretion in influenza A virus infected human bronchial epithelial cells. J Ethnopharmacol 2005;102:418-423.

20 Kim S.H, Jang YP, Sung SH, Kim YC: Inhibitory activity of phenolic glycosides from the fruits of Idesia polycarpa on lipopolysaccharide-induced nitric oxide production in BV2 microglia. Planta Med 2007;73:167-169.

21 Ozaki Y, Rui J, Tang YT: Antiinflammatory effect of Forsythia Suspensa Vahl and its active principle. Biol Pharm Bull 2000;23:365-367.

22 Kim MS, Na H J, Han SW, Jin JS, Song UY, Lee EJ, Song BK, Hong SH, Kim HM.: Forsythia fructus inhibits the mast-cell-mediated allergic inflammatory reactions. Inflammation 2003;27:129-135.

23 Guo H, Liu AH, Li L, Guo DA: Simultaneous determination of 12 major constituents in Forsythia suspensa by high performance liquid chromatography_DADmethod. J Pharm Biomedi Anal 2007a;43:1000-1006.

24 Wang L, Piao XL, Kim SW, Piao XS, Shen YB, Lee HS: Effects of Forsythia suspensa extract on growth performance, nutrient digestibility, and antioxidant activities in broiler chickens under high ambient temperature. Poult Sci 2008;87:1287-1294.

25 Piao XL, Jang MH, Cui J, Piao XS: Lignans from the fruits of Forsythia suspensa. Bioorg Med Chem Lett 2008;18:1980-1984.

26 Qu H,Zhang Y, Wang Y, Li B, Sun W: Antioxidant and antibacterial activity of two compounds (forsythiaside and forsythin) isolated from forsythia suspensa. J Pharm Pharmacol 2008;60:261-265.

27 Do MT, Kim HG, Choi JH, Khanal T, Park BH, Tran TP, Hwang YP, Na M, Jeong HG: Phillyrin attenuates high glucose-induced lipid accumulation in human HepG2 hepatocytes through the activation of LKB1/AMPactivated protein kinase-dependent signaling. Food Chem 2013;136:415-425.

28 Díaz Lanza AM, Abad Martínez MJ, Fernández Matellano L, Recuero Carretero C, Villaescusa Castillo L, Silván Sen AM, Bermejo Benito P: Lignan and Phenylpropanoid Glycosides from Phillyrea latifolia and their In vitro Anti-Inflammatory Activity. Planta Med 2001;67:219-223

29 Lee DG, Lee SM, Bang MH, Park HJ, Lee TH, Kim YH, Kim JY, Baek NI: Lignans from the flowers of Osmanthus fragrans var. aurantiacus and their inhibition effect on NO production. Arch Pharm Res 2011;34:2029-2035.

30 Westerfield M: The zebrafish book. A guide for the laboratory use of zebrafish (Danio rerio) M. Univ of Oregon Press, Eugene 4th ed. Oregon, 2007, pp4-20

-31 Yang LL, Wang GQ, Yang LM, Huang ZB, Zhang WQ, Yu LZ: Endotoxin Molecule Lipopolysaccharide- Induced Zebrafish Inflammation Model: A Novel Screening Method for Anti-Inflammatory Drugs. Molecules 2014;19:2390-2409.

-32 Guyader DL, Redd MJ, Colucci-Guyon E: Origins and unconventional behavior of neutrophils in developing zebrafish. J Blood 2008;111:132-41.

-33 Silva WFD, Egami MI, Santos AA, Antoniazzi MM, Silva M, Gutierre RC, Paiva MJR: Cytochemical, immunocytochemical and ultrastructural observations on leukocytes and thrombocytes of fat snook (Centropomus parallelus). Fish Shellfish Immun 2011;31:571-577.

-34 Novoa B, Figueras A: Zebrafish: model for the study of inflammation and the innate immune response to infectious diseases. Adv Exp Med Biol 2012;946:253-275.

-35 Zhang G, Ghosh S: Toll-like receptor-mediated NF-kappaB activation: a phylogenetically conserved paradigm in innate immunity. J Clin Invest 2001;107:13-19. 


\section{Cellular Physiology Cell Physiol Biochem 2017;43:2074-2087 \begin{tabular}{l|l|l}
\hline and Biochemistry $10.1159 / 000484192$ & $\begin{array}{l}\text { C } 2017 \text { The Author(s). Published by S. Karger AG, Basel } \\
\text { www.karger.com/cpb }\end{array}$ \\
\hline
\end{tabular}}

Yang et al.: Protective Effect of PHN in Zebrafish

36 Bonizzi G, Karin M: The two NF-kappaB activation pathways and their role in innate and adaptive immunity. Trends Immunol 2004;25:280-288.

-37 Chen LP, Liu C, Chiang FY, Wang LF, Lee KW, Chen WT, Kuo PL, Liang CH: IL-8 promotes inflammatory mediators and stimulates activation of p38 MAPK/ERK-NF-kB pathway and reduction of JNK in HNSCC. Oncotarget.2017, Doi:10.18632 / oncotarget.16914

-38 Carter AB, Monick MM, Hunninghake GW: Both Erk and p38 kinases are necessary for cytokine gene transcription. Am J Respir Cell Mol Bio 1999;20:751-758

-39 Qi S, Xin Y, Guo Y, Diao Y, Kou X, Luo L, Yin Z: Ampelopsin reduces endotoxic inflammation via repressing ROS-mediated activation of PI3 K/Akt/NF-j B signaling pathways. Int Immunopharmacol 2012;12:278287.

40 Li B, Zhang R, Li J, Zhang L,Ding G,Luo P,He S,Dong Y,Jiang W,Lu Y,Cao H, Zheng J,Zhou H: Antimalarial artesunate protects sepsis model mice against heat-killed Escherichia coli challenge by decreasing TLR4, TLR9 mRNA expressions and transcription factor NF-кB activation. Int Immunopharmacology 2008;8:379389

41 Cheng BC, Ma XQ, Kwan HY, Tse KW,Cao HH,Su T,Shu X, Wu ZZ, Yu ZL: A herbal formula consisting of Rosae Multiflorae Fructus and Lonicerae Japonicae Flos inhibits inflammatory mediators in LPS-stimulated RAW 264.7 macrophages. J Ethnopharmacol 2014;153:922-927.

42 Liu JS, Wei XD, Lu ZB, Xie P,Zhou HL: Liang-Ge-San, a classic traditional Chinese medicine formula, protects against lipopolysaccharide-induced inflammation through cholinergic anti-inflammatory pathway. Oncotarget 2016;7:21222-21234.

-43 Berger J, Currie PD: Zebrafish models flex their muscles to shed light on muscular dystrophies. Dis Model Mech 2012;5:726-732.

44 Jiang JX, Yao YM, Zheng J: The basic and clinical of bacterial endotoxin. People's Military Medical Press, Beijing, 2004, pp194-195.

45 Hayashi F, Means TK, Luster AD: Toll-like receptor stimulate human neutrophil function. Blood 2003;102:2660-2669.

46 Zhang S, Rahman M, Zhang, S, Qi Z,Thorlacius H: Simvastatin antagonizes CD40L secretion, CXC chemokine for mation, and pulmonary infiltration of neutrophils in abdominal sepsis. J Leukoc Biol 2011;89:735-742.

-47 Elphick GF, Sarangi PP, Hyun YM, Hollenbaugh JA, Ayala A, Biffl WL, Chung HL, Rezaie AR, McGrath JL, Topham DJ, Reichner JS, Kim M: Recombi-nant human activated protein C inhibits integrin-medi-ated neutrophil migration. Blood 2009;113:4078-4085.

48 Asaduzzaman M, Lavasani S, Rahman M, Zhang S, Braun O0, Jeppsson B, Thorlacius H: Platelets support pulmonary recruitment of neutrophils in abdominalsepsis. Critl Care Med 2009;37:1389-1396.

49 Wei YM, Liao YH, Chu SJ, Zhou QJ, Wang GR, WANG CH, Cheng LX: Role of apoptosis of neutrophis and vascular endothelial cells and changes of relative factors in the pathogenesis of traumatic sepsis at the advanced stage. Chin J Trauma 2006;9:686-689.

-50 Lekstrom-Himes JA, Gallin JI, Immunodeficiency diseases caused by defects in phagocytes. N Engl J Med 2000;343:1703-1714

-51 Moreno GM: Neutropenia in HIV infection. An Med Intera 1997;14:199-208.

52 Liu J: Experimental study on the immunological function and anti-stress of phillyrin. J Shanxi Ins Edu 2008;24:59-61.

53 Ghosh S,Hayden MS: New regulators of NF-kappaB in inflammation. Nat Rev Immunol 2008;8:837-848.

$>54$ Schuh K, Pahl A: Inhibition of the map kinase erk protects from lipopolysaccharide-induced lung injury. Biochem pharmacol 2009;77:1827-1834.

55 Zhong WT, Wu YC, Xie XX, Zhou X, Wei MM, Soromou LW, Ci XX, Wang DC: Phillyrin attenuates lps-induced pulmonary inflammation via suppression of mapk and nf-kappab activation in acute lung injury mice. Fitoterapia 2013;90:132-139. 